\title{
Ground state of a trapped Bose-Einstein condensate in two dimensions: Beyond the mean-field approximation
}

\author{
Jens O. Andersen \\ Institute for Theoretical Physics, University of Utrecht, Leuvenlaan 4, 3584 CE Utrecht, The Netherlands \\ Hårek Haugerud \\ Faculty of Engineering, Oslo University College, Cort Adelers gate 30, 0254 Oslo, Norway
}

(Received 17 September 2001; published 20 February 2002)

\begin{abstract}
We consider the ground state of a trapped Bose-Einstein condensate in two dimensions. In the mean-field approximation the ground-state density profile satisfies the Gross-Pitaevskii equation. We compute the leading quantum corrections to the density profile to second order in an expansion around the Thomas-Fermi limit. By summing the ladder diagrams, we obtain a modified equation that includes gradient corrections.
\end{abstract}

DOI: 10.1103/PhysRevA.65.033615

\section{INTRODUCTION}

The remarkable achievement of Bose-Einstein condensation (BEC) of alkali-metal atoms in harmonic traps [1-3] has created an enormous interest in the properties of dilute Bose gases (see, e.g., Ref. [4] and references therein). One of the basic questions is the condensate density profile as a function of temperature. At zero temperature and in the mean-field approximation, the condensate density satisfies the GrossPitaevskii equation. Until recently, the condensates were so dilute that mean-field theory gives a satisfactory description of the experiments. However, there are corrections from quantum fluctuations around the mean field and their relative importance grows with the gas parameter $\sqrt{\rho a^{3}}$, where $a$ is the $s$-wave scattering length and $\rho$ is the density. The sign and value of the scattering length are determined by the nature and the strength of the interatomic interaction and the scattering length can be manipulated by an external field. In recent experiments, Cornish et al. [5] were able to vary the scattering length $a$ over a large range by applying a strong external magnetic field and exploiting the existence of a Feshbach resonance in ${ }^{85} \mathrm{Rb}$ at $B \sim 155 \mathrm{G}$. Values for $\sqrt{\rho a^{3}}$ up to approximately 0.1 were obtained and should be sufficiently large to see deviations from the mean field in experiments. Hence it becomes important to be able to calculate the effects of quantum fluctuations around the mean field in a systematic way. Such an approach was developed in Ref. [6], where the effects of quantum fluctuations on the ground state of a Bose-Einstein condensate in three dimensions were calculated. In Ref. [7], the result was extended to arbitrary timeindependent states, including vortices. The method is a combination of the Hartree-Fock approach and the ThomasFermi approach. The Hartree-Fock method includes all leading quantum corrections to the mean-field equation for the density. The resulting equation is an integral equation, which is reduced to a local differential equation by applying a gradient expansion around the Thomas-Fermi limit.

In a recent paper, Blume and Greene [8] use a diffusion Monte Carlo method to calculate the ground-state energy for different two-body potentials that generate the same value for the scattering length $a$. It turns out that the ground-state energy is independent of the actual form of the potential, it
PACS number(s): 03.75.Fi

only depends on $a$. Moreover, it differs significantly from the ground-state energy obtained from solving the GrossPitaevskii equation. Including the leading quantum corrections to zeroth order in a gradient expansion, the agreement with the result obtained by Blume and Greene [8] improves significantly.

The ground-state energy density of a two-dimensional homogeneous Bose gas was first calculated by Schick [9]. By summing up the ladder diagrams contributing to the chemical potential, he showed that, in the dilute limit, the bare interaction $g$ is replaced by an effective interaction $\sim\left[\ln \left(\rho a^{2}\right)\right]^{-1}$, where $\rho$ is the density and $a$ is the range of the interaction. This parameter is the two-body $T$ matrix, and Schick determined the leading-order result in $T$. The next-toleading order result has later been derived by several authors $[10-14]$. Very recently, a formal proof of the result by Schick was given by Lieb and co-workers $[15,16]$.

It is well known that a Bose-Einstein condensate in a two-dimensional homogeneous Bose gas only exists at $T$ $=0$. At finite temperature, phase fluctuations destroy the condensate. This reflects the Mermin-Wagner theorem [17,18] stating that there is no spontaneous breakdown of a continuous symmetry in a homogeneous system in two dimensions at finite temperature. The physics of two-dimensional homogeneous Bose gases is nonetheless very interesting. A dilute homogeneous Bose gas in two dimensions is expected to undergo a phase transition at finite temperature, which is the Kosterlitz-Thouless transition [18]. Below the critical temperature, the gas is superfluid but has only algebraic longrange order. This topological phase transition is not characterized by a local order parameter, but by the unbinding of vortex pairs and the destruction of superfluidity. The superfluid phase is characterized by the existence of a so-called quasicondensate [11], which roughly speaking corresponds to a condensate with a fluctuating phase. The existence of a quasicondensate has only very recently been observed in spin-polarized hydrogen adsorbed on a superfluid ${ }^{47} \mathrm{He}$ surface by Safonov et al. [20].

The Mermin-Wagner theorem does not apply to an inhomogeneous system and a condensate may exist. Bagnato and Kleppner [21] showed the possibility of BEC for $T<T_{c}$ $\approx \sqrt{N} \hbar \omega$ (where $N$ is the number of particles and $\omega$ is the 
trap frequency) in a two-dimensional ideal gas confined by a harmonic potential. In this temperature range, the phase fluctuations on the scale of the size of a trapped gas is negligible, and there is a true condensate. In Refs. [22,23], it was shown that the possibility of BEC exists also when local two-body interactions are included (see also Ref. [24]).

Low-dimensional trapped Bose gases are becoming of increasing interest theoretically as they are now being realized experimentally. In a very recent experiment by Görlitz et al. [25], one and two-dimensional Bose-Einstein condensates were created by reducing the temperature and the average interaction energy of the atoms below the energy splitting of either two or one of the directions of the harmonic trapping potential, respectively. For relation to other possible experiments, see, e.g., Refs. [4,26].

The ground-state properties of a trapped Bose gas in two dimensions have been studied recently by a number of authors. In Refs. [27-29], the Gross-Pitaevskii equation was solved numerically using the bare coupling $g$. In Refs. $[15,16,30]$, it is argued that the correct equation for the density can be derived from an energy functional that contains the result for the energy density obtained by Schick [9] and therefore involves a density-dependent coupling.

However, the Gross-Pitaevskii equation is a mean-field equation and receives quantum corrections, and in the case of trapped Bose gases these involve gradients of the density. In the present paper, we calculate the leading quantum corrections to the Gross-Pitaevskii equation to second order in the gradient expansion around the Thomas-Fermi limit. We also apply the $T$-matrix approximation to sum up the ladder diagrams. The resulting equation extends the result in Refs. $[15,16,30]$.

The plan of the paper is as follows. In Sec. II, we review the perturbative framework developed in Ref. [6] to calculate the leading quantum corrections to the Gross-Pitaevskii equation. In Sec. III, we calculate the self-consistent oneloop corrections to second order in the gradient expansion. In Sec. IV, we briefly discuss the summation of the ladder diagrams and derive an equation that takes this summation into account. Finally, we summarize our results in Sec. V. Calculational details are included in two appendixes.

\section{PERTURBATIVE FRAMEWORK}

In this section, we discuss the perturbative framework developed in Ref. [6] to calculate the leading effects on the ground state from quantum fluctuations around the mean field.

The action is

$$
\begin{aligned}
S[\psi]= & \int d t\left\{\int d^{2} x \psi^{*}(\mathbf{x}, t)\left[i \hbar \frac{\partial}{\partial t}+\frac{\hbar^{2}}{2 m} \nabla^{2}+\mu-V(\mathbf{x})\right]\right. \\
& \times \psi(\mathbf{x}, t)-\frac{1}{2} \int d^{2} x \int d^{2} x^{\prime} \psi^{*}(\mathbf{x}, t) \psi^{*}\left(\mathbf{x}^{\prime}, t\right) \\
& \left.\times V_{0}\left(\mathbf{x}-\mathbf{x}^{\prime}\right) \psi(\mathbf{x}, t) \psi\left(\mathbf{x}^{\prime}, t\right)\right\}
\end{aligned}
$$

$\psi^{*}(\mathbf{x}, t)$ is a complex field operator that creates a boson at the position $\mathbf{x} . \mu$ is the chemical potential, $V(\mathbf{x})$ is the external trapping potential, $V_{0}(\mathbf{x})$ is the two-body potential. In the following, we set $\hbar=2 m=1$. Factors of $\hbar$ and $2 m$ can be reinserted using dimensional analysis.

The action (1) is symmetric under a phase transformation

$$
\psi(\mathbf{x}, t) \rightarrow e^{i \alpha} \psi(\mathbf{x}, t)
$$

This U(1) symmetry ensures that the density $\rho$ and current density $\mathbf{j}$ satisfy the continuity equation

$$
\dot{\rho}+\nabla \cdot \mathbf{j}=0 .
$$

In the ground state, the current density $\mathbf{j}$ vanishes identically and the condensate has a constant phase. The U(1) symmetry can then be used to make the condensate real everywhere.

If the interatomic potential $V_{0}(\mathbf{x})$ is short range, it can be mimicked by local interactions. If the energies are low enough, the scattering amplitude can be approximated by $s$-wave scattering and the action Eq. (1) can be approximated by [31]

$$
S[\psi]=\int d t \int d^{2} x \psi^{*}\left[i \frac{\partial}{\partial t}+\nabla^{2}+\mu-V\right] \psi-\frac{1}{2} g\left(\psi^{*} \psi\right)^{2} .
$$

The quantum field theory defined by the action (4) has ultraviolet divergences that must be removed by renormalization of $\mu$ and $g$. There is also an ultraviolet divergence in the expression for the density $\rho$. This divergence can be removed by adding a counterterm $\delta \rho$. Alternatively, one can eliminate the divergences associated with $\mu$ and $\rho$ by a normal-ordering prescription of the fields in Eq. (4). The coupling constant is renormalized in the usual way by replacing the bare coupling with the physical one.

If we use a simple momentum cutoff $M$ to cut off the ultraviolet divergences in the loop integrals, there will be terms proportional to $M^{p}$, where $p$ is a positive integer. There are also terms that are proportional to $\ln (M)$. The coefficients of the power divergences depend on the regularization method and are therefore artifacts of the regulator. On the other hand, the coefficients of $\ln (M)$ are independent of the regulator and they therefore represent real physics. In this paper, we use dimensional regularization to regulate both infrared and ultraviolet divergences. In dimensional regularization, one calculates the loop integrals in $d=2-2 \epsilon$ dimensions for values of $\epsilon$ where the integrals converge. One then analytically continues back to $d=2$ dimensions. With dimensional regularization, an arbitrary renormalization scale $M$ is introduced. This scale can be identified with the simple momentum cutoff mentioned above. An advantage of dimensional regularization is that it automatically sets power divergences to zero, while logarithmic divergences show up as poles in $\epsilon$. In two dimensions, the one-loop counterterms for the chemical potential $\mu$ and the density $\rho$ are quadratic ultraviolet divergences, while the one-loop counterterm for the coupling constant $g$ is a logarithmic ultraviolet divergence. 
We next parametrize the quantum field $\psi$ in terms of a time-independent condensate $v$ and a quantum fluctuating field $\widetilde{\psi}$

$$
\psi=v+\widetilde{\psi}
$$

where the condensate $v$ satisfies

$$
v=\langle\psi\rangle \text {. }
$$

Here and in the following, $\langle A\rangle$ denotes expectation value of the operator $A$ in the ground state. Thus the expectation value of $\widetilde{\psi}$ vanishes. The fluctuating field can be written in terms of two real fields,

$$
\widetilde{\psi}=\frac{1}{\sqrt{2}}\left(\psi_{1}+i \psi_{2}\right) .
$$

Substituting Eq. (7) into Eq. (4), the action can be decomposed into three terms,

$$
S[\psi]=S[v]+S_{\text {free }}\left[\psi_{1}, \psi_{2}\right]+S_{\text {int }}\left[v, \psi_{1}, \psi_{2}\right] .
$$

$S[v]$ is the classical action

$$
S[v]=\int d t \int d^{2} x\left[(\mu-V) v^{2}-\frac{1}{2} g v^{4}+v \nabla^{2} v\right],
$$

while the free part of the action is

$$
\begin{aligned}
S_{\text {free }}\left[\psi_{1}, \psi_{2}\right]= & \int d t \int d^{2} x\left[\frac{1}{2}\left(\psi_{1} \psi_{2}-\psi_{1} \psi_{2}\right)\right. \\
& \left.+\frac{1}{2} \psi_{1}\left(\nabla^{2}-\Lambda^{2}\right) \psi_{1}+\frac{1}{2} \psi_{2} \nabla^{2} \psi_{2}\right] .
\end{aligned}
$$

The interaction part of the action is

$$
\begin{aligned}
S_{\text {int }}\left[v, \psi_{1}, \psi_{2}\right]= & \int d t \int d^{2} x\left[\sqrt{2} T \psi_{1}+\frac{1}{2} X \psi_{1}^{2}+\frac{1}{2} Y \psi_{2}^{2}\right. \\
& \left.+\frac{1}{\sqrt{2}} Z \psi_{1}\left(\psi_{1}^{2}+\psi_{2}^{2}\right)-\frac{1}{8} g\left(\psi_{1}^{2}+\psi_{2}^{2}\right)^{2}\right] .
\end{aligned}
$$

The sources in Eq. (11) are

$$
\begin{gathered}
T=\left[\mu-V-g v^{2}\right] v+\nabla^{2} v, \\
X=\Lambda^{2}+\left[\mu-V-3 g v^{2}\right], \\
Y=\left[\mu-V-g v^{2}\right], \\
Z=-g v .
\end{gathered}
$$

Note that we have added and subtracted an arbitrary term $\frac{1}{2} \Lambda^{2} \psi_{1}^{2}$ in the action. Its effects are subtracted out at higher orders through the source $X$. By a judicious choice of $\Lambda$, one can ensure that $X$ can be treated as a perturbation in the same way as the other sources and thus simplify calculations. We return to the choice of $\Lambda$ in the following section.
The propagator (evaluated at the classical minimum) that corresponds to the free action $S_{\text {free }}\left[\psi_{1}, \psi_{2}\right]$ in Eq. (10) is

$$
D(\omega, k)=\frac{i}{\omega^{2}-\epsilon^{2}(k)+i \epsilon}\left(\begin{array}{cc}
k^{2} & -i \omega \\
i \omega & \epsilon^{2}(k) / k^{2}
\end{array}\right) .
$$

Here $\mathbf{k}$ is the wave vector, $\omega$ is the frequency, and $\epsilon(k)$ is the dispersion relation for the Bogoliubov modes

$$
\epsilon(k)=k \sqrt{k^{2}+\Lambda^{2}} .
$$

The dispersion relation is gapless, which reflects the spontaneous breakdown of the U(1) symmetry (Goldstone's theorem). The dispersion is quadratic for large wave vectors and is that of a free nonrelativistic particle. The propagator is defined with an $i \epsilon$ prescription in the usual way. The quantum field theory defined by Eq. (4) has been decomposed into free and interacting parts. The quantum loop expansion is then an expansion in the dimensionless coupling constant $g$. The Hartree-Fock approximation includes all effects to leading order in $g$.

The field equation for $\psi_{1}$ (and $\psi_{2}$ ) is obtained by varying the action (8). Taking the expectation value of the equation for $\psi_{1}$, we obtain the tadpole equation

$$
0=T+\frac{3}{2} Z\left\langle\psi_{1}^{2}\right\rangle+\frac{1}{2} Z\left\langle\psi_{2}^{2}\right\rangle-\frac{1}{2 \sqrt{2}} g\left\langle\psi_{1}\left(\psi_{1}^{2}+\psi_{2}^{2}\right)\right\rangle .
$$

The density can be written as

$$
\rho=\left\langle\psi^{*} \psi\right\rangle=v^{2}+\frac{1}{2}\left\langle\psi_{1}^{2}\right\rangle+\frac{1}{2}\left\langle\psi_{2}^{2}\right\rangle
$$

To zeroth order in the loop expansion, one neglects the expectation values in Eq. (18) and the tadpole equation reduces to $T=0$. We also neglect the expectation values in Eq. (19) and we have $\rho=v^{2}$. The tadpole equation then reduces to the Gross-Pitaevskii equation for the density profile

$$
[\mu-V-g \rho] \sqrt{\rho}+\nabla^{2} \sqrt{\rho}=0 .
$$

The last term in Eq. (18) only contributes at second order and beyond in the loop expansion. Thus to leading order in the quantum fluctuations, Eq. (18) reduces to

$$
0=T+\frac{3}{2} Z\left\langle\psi_{1}^{2}\right\rangle+\frac{1}{2} Z\left\langle\psi_{2}^{2}\right\rangle .
$$

Equation (21) is referred to as the semiclassical tadpole equation [6].

The sources $X$ and $Y$ depend on the condensate $v$. In order to obtain an equation for the density $\rho$, we invert Eq. (19) so that we can eliminate $v$ in favor of $\rho$ in Eq. (21). The expectation values in Eq. (19) are correction terms arising from quantum fluctuations around the mean field. These terms are suppressed by powers of $g$ compared to the terms in the classical equations. Since we are only interested in the leading quantum effects, we can invert Eq. (19) and expand it to first order in the expectation values,

$$
v=\sqrt{\rho}-\frac{1}{4 \sqrt{\rho}}\left\langle\psi_{1}^{2}+\psi_{2}^{2}\right\rangle .
$$


We can also derive expressions for the gradients of $v$ by differentiating Eq. (22),

$$
\begin{aligned}
\boldsymbol{\nabla} v= & \frac{1}{4 \rho}\left[4 \rho \boldsymbol{\nabla} \sqrt{\rho}+\boldsymbol{\nabla} \sqrt{\rho}\left\langle\psi_{1}^{2}+\psi_{2}^{2}\right\rangle-\sqrt{\rho} \boldsymbol{\nabla}\left\langle\psi_{1}^{2}+\psi_{2}^{2}\right\rangle\right], \\
\nabla^{2} v= & \frac{1}{4 \rho^{3 / 2}}\left[4 \rho^{3 / 2} \nabla^{2} \sqrt{\rho}+\left[\sqrt{\rho} \nabla^{2} \sqrt{\rho}-2(\boldsymbol{\nabla} \sqrt{\rho})^{2}\right]\left\langle\psi_{1}^{2}+\psi_{2}^{2}\right\rangle\right. \\
& \left.+2 \sqrt{\rho} \boldsymbol{\nabla} \sqrt{\rho} \cdot \boldsymbol{\nabla}\left\langle\psi_{1}^{2}+\psi_{2}^{2}\right\rangle-\rho \nabla^{2}\left\langle\psi_{1}^{2}+\psi_{2}^{2}\right\rangle\right] .
\end{aligned}
$$

Substituting the expression (22) for $v$ and Eq. (24) for $\nabla^{2} v$, the semiclassical tadpole equation (21) becomes

$$
\begin{aligned}
0= & {[\mu-V-g \rho] \sqrt{\rho}+\nabla^{2} \sqrt{\rho}-g \sqrt{\rho}\left\langle\psi_{1}^{2}\right\rangle+\frac{1}{2 \rho^{3 / 2}}\left[\sqrt{\rho} \nabla^{2} \sqrt{\rho}\right.} \\
& \left.-(\nabla \sqrt{\rho})^{2}\right]\left\langle\psi_{1}^{2}+\psi_{2}^{2}\right\rangle+\frac{1}{2 \rho} \nabla \sqrt{\rho} \cdot \nabla\left\langle\psi_{1}^{2}+\psi_{2}^{2}\right\rangle \\
& -\frac{1}{4 \sqrt{\rho}} \nabla^{2}\left\langle\psi_{1}^{2}+\psi_{2}^{2}\right\rangle-\frac{1}{4 \sqrt{\rho}}\left(\mu-V-g \rho+\frac{1}{\sqrt{\rho}} \nabla^{2} \sqrt{\rho}\right) \\
& \times\left\langle\psi_{1}^{2}+\psi_{2}^{2}\right\rangle .
\end{aligned}
$$

The last line in Eq. (25) is proportional to the classical equation of motion. The corrections to the classical equation of motion are of order $g$ and so are the expectation values of the quantum fields. Thus the last line is second order in quantum corrections and will therefore be dropped in the following.

\section{GRADIENT EXPANSION}

The expectation values in Eq. (21) are functionals of the sources $X$ and $Y$. These functionals involve an arbitrary number of insertions of the sources in the loop diagrams for $\left\langle\psi_{1}^{2}\right\rangle$ and $\left\langle\psi_{2}^{2}\right\rangle$. They are nonlocal since the positions of the sources are integrated over. Thus the tadpole equation is an integral equation.

Instead of solving the nonlocal integral equation (25), we would like to derive a local differential equation by expanding the expectation values $\left\langle\psi_{1}^{2}\right\rangle$ and $\left\langle\psi_{2}^{2}\right\rangle$ in powers of the sources and their gradients. The Thomas-Fermi expansion is an expansion in powers of $\xi / R$, where $\xi=1 / \sqrt{g \rho}$ is the local coherence length and $R$ is the length scale for significant changes in the density. It is possible to expand the expectation values in Eq. (21) in powers of gradients of the sources, if the expectation values receive significant contributions only from modes with wavelengths that are of the order of the coherence length or less. This can be ensured by introducing an infrared cutoff that eliminates the contribution from wavelengths larger than the coherence length. We use dimensional regularization to guarantee that the effects of these modes are eliminated. If the results depend on the infrared cutoff, it indicates a sensitivity to length scales much larger than the coherence length [6].

We can also expand the expectation values in powers of $X$ and $Y$ if the sources are at least first order either in the gra-

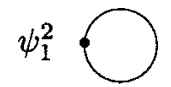

FIG. 1. Feynman diagram needed for the calculation of the coefficient of $a_{0}$ in Eq. (29).

dient expansion or in the coupling constant $g$. This is generally not correct, but it can be made true at a specific point $\mathbf{x}_{0}$ by a clever choice of the parameter $\Lambda$ [6]. Since the expectation values of the quantum fields are of order $g$ and we are interested in the leading quantum effects, we can use the classical equation of motion, $T=0$, to simplify the sources,

$$
\begin{gathered}
X\left(\mathbf{x}_{0}\right)=\Lambda^{2}-2 g v^{2}-\frac{1}{v} \nabla^{2} v, \\
Y\left(\mathbf{x}_{0}\right)=-\frac{1}{v} \nabla^{2} v .
\end{gathered}
$$

The source $Y\left(\mathbf{x}_{0}\right)$ is already second order in the gradient expansion. We can also make $X\left(\mathbf{x}_{0}\right)$ second order in the gradient expansion by the following choice [6] for the arbitrary parameter $\Lambda$ :

$$
\Lambda^{2}=2 g \rho\left(\mathbf{x}_{0}\right) .
$$

The difference between $\rho$ and $v^{2}$ is higher order in $g$ and can be neglected. Any other choice of $\Lambda$, which is equal to Eq. (28) to leading order in $g$ and in the gradient expansion, results in the same final equation for the density.

The expectation values in Eq. (25) are now expanded in powers of $X$ and $Y$ and their gradients around the point $\mathbf{x}_{0}$,

$$
\begin{aligned}
& \left\langle\psi_{1}^{2}\right\rangle=a_{0}+a_{1} X+a_{2} Y+a_{3} \nabla^{2} X+a_{4} X^{2}+a_{5}(\nabla X)^{2}+\cdots \\
& \left\langle\psi_{2}^{2}\right\rangle=b_{0}+b_{1} X+b_{2} Y+b_{3} \nabla^{2} X+b_{4} X^{2}+b_{5}(\nabla X)^{2}+\cdots
\end{aligned}
$$

The expansions of $\left\langle\psi_{1}\right\rangle$ and $\left\langle\psi_{2}\right\rangle$ include all rotationally invariant terms built from the sources and their derivatives. In Fig. 1, we show the diagram that we need to calculate in order to obtain the coefficient $a_{0}$ appearing in Eq. (29). The solid line denotes the diagonal propagator for $\psi_{1}$. The calculation is sketched in Appendix B.

We will also need to calculate the gradients of $\left\langle\psi_{1}^{2}\right\rangle$ and $\left\langle\psi_{2}^{2}\right\rangle$ in powers of the sources and their derivatives. Adding Eqs. (29) and (30) and differentiating, we obtain

$$
\begin{gathered}
\boldsymbol{\nabla}\left\langle\psi_{1}^{2}+\psi_{2}^{2}\right\rangle=\left(a_{1}+b_{1}\right) \nabla X+\cdots \\
\nabla^{2}\left\langle\psi_{1}^{2}+\psi_{2}^{2}\right\rangle=\left(a_{1}+b_{1}\right) \nabla^{2} X+2\left(a_{4}+b_{4}\right)(\nabla X)^{2}+\cdots
\end{gathered}
$$

We next eliminate the dependence on $v$ of the sources in favor of the density $\rho$. Since the sources only appear in the expectation values that are of order $g$, we can neglect the expectation values in Eqs. (22)-(24),

$$
v\left(\mathbf{x}_{0}\right)=\sqrt{\rho},
$$




$$
\begin{gathered}
\boldsymbol{\nabla} v\left(\mathbf{x}_{0}\right)=\nabla \sqrt{\rho}, \\
\nabla^{2} v\left(\mathbf{x}_{0}\right)=\nabla^{2} \sqrt{\rho} .
\end{gathered}
$$

The resulting expressions for the sources and their derivatives then become

$$
\begin{gathered}
X\left(\mathbf{x}_{0}\right)=-\frac{\nabla^{2} \sqrt{\rho}}{\sqrt{\rho}}, \\
\nabla X\left(\mathbf{x}_{0}\right)=-4 g \sqrt{\rho} \nabla \sqrt{\rho}, \\
\nabla^{2} X\left(\mathbf{x}_{0}\right)=-4 g\left[\sqrt{\rho} \nabla^{2} \sqrt{\rho}+(\nabla \sqrt{\rho})^{2}\right], \\
Y\left(\mathbf{x}_{0}\right)=-\frac{\nabla^{2} \sqrt{\rho}}{\sqrt{\rho}} .
\end{gathered}
$$

Using Eqs. (29)-(32) for the expectation values and the expressions (36)-(39) for the sources, the semiclassical tadpole equation reduces to

$$
\begin{aligned}
0= & (\mu-V-g \rho) \sqrt{\rho}+\nabla^{2} \sqrt{\rho}-a_{0} g \sqrt{\rho}+\frac{1}{2}\left[a_{0}+b_{0}+\left(2 a_{1}\right.\right. \\
& \left.\left.+b_{1}+a_{2}\right) \Lambda^{2}+2 a_{3} \Lambda^{4}\right] \frac{\nabla^{2} \sqrt{\rho}}{\rho}-\frac{1}{2}\left[a_{0}+b_{0}+\left(a_{1}+b_{1}\right) \Lambda^{2}\right. \\
& \left.-2\left(a_{3}-2 a_{4}-2 b_{4}\right) \Lambda^{4}+4 a_{5} \Lambda^{6}\right] \frac{(\nabla \sqrt{\rho})^{2}}{\rho \sqrt{\rho}}
\end{aligned}
$$

The coefficients $a_{i}$ and $b_{i}$ were calculated in Ref. [6]. They can be expressed in terms of the integral $I_{m, n}$, which is defined in Appendix A. We list them in Appendix B for convenience. The coefficients $a_{0}$ and $b_{0}$ are quadratically ultraviolet divergent, while $a_{1}, a_{2}, b_{1}$, and $b_{2}$ are logarithmically ultraviolet divergent. The remaining coefficients are ultraviolet finite. Note also that the infrared divergences in the individual coefficients cancel in the sum. The fact that the dependence on the infrared cutoff cancels, ensures that there is no sensitivity to length scales much larger than the coherence length to leading order in the gradient expansion.

The counterterm needed to cancel the ultraviolet divergences in Eq. (40) is [32]

$$
\delta g=\frac{g^{2}}{8 \pi \epsilon} .
$$

Using the values for the coefficients listed in Appendix B, we obtain

$$
\begin{aligned}
0= & {\left[\nabla^{2}+\mu-V\right] \sqrt{\rho}-g \rho \sqrt{\rho}-\frac{g^{2}}{8 \pi}\left\{1+\ln \left[\frac{g \rho}{2 M^{2}}\right]\right\} \rho \sqrt{\rho} } \\
& -\frac{g}{12 \pi} \nabla^{2} \sqrt{\rho}
\end{aligned}
$$

Note, in particular, that the coefficient of $(\nabla \sqrt{\rho})^{2}$ in Eq. (40) vanishes. This is not the case in three dimensions [6] and we have no explanation for this cancellation. Equation (42) has

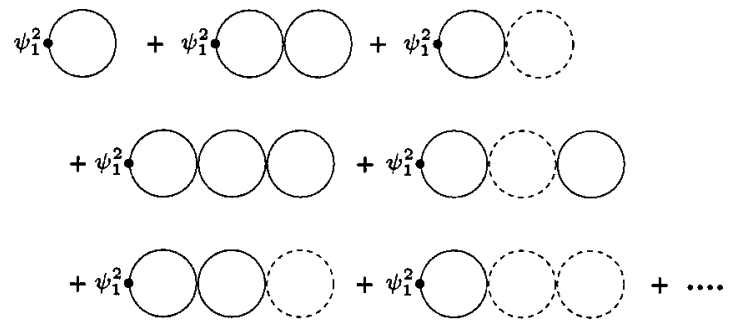

FIG. 2. Series of diagrams that are summed to give the leading correction to the mean-field term $g \rho \sqrt{\rho}$ in Eq. (42).

been derived at a specific point $\mathbf{x}_{0}$. Since the point $\mathbf{x}_{0}$ is arbitrary, Eq. (42) must hold everywhere.

The logarithmic term in Eq. (42) can be obtained from the one-loop result for the ground-state energy density $\mathcal{E}$ for a homogeneous Bose gas, obtained in Refs. [33,34] by differentiating $\mathcal{E}$ with respect to $\sqrt{\rho}$. The last term in Eq. (42) is a new result.

This renormalization scale is completely arbitrary and physical quantities such as the density profile of the ground state must be independent of it. The requirement that physical quantities be independent of $M$ can be expressed in terms of renormalization-group equations for the coupling constants in the Lagrangian. The coupling constant $g$ in Eq. (4) satisfies

$$
M \frac{d}{d M} g=\beta(g)
$$

where the $\beta$ function is a polynomial in $g$. Normally, $\beta$ functions are known only up to a certain order in the quantum loop expansion. In the present case, the one-loop result for the $\beta$ function is exact and $\beta(g)=g^{2} / 4 \pi[32]$.

\section{IV. $T$-MATRIX APPROXIMATION}

In this section we employ the ladder or $T$-matrix approximation, which takes an infinite number of loops into account.

The diagrams we are summing and that give the leading correction to the mean-field term $g \rho \sqrt{\rho}$ in Eq. (42) are shown in Fig. 2. The solid line denotes the diagonal propagator for $\psi_{1}$ and the dashed line denotes the diagonal propagator for $\psi_{2}$. The off-diagonal propagators for $\psi_{1}$ and $\psi_{2}$ are denoted by a solid-dashed and dashed-solid line, respectively. The diagrams are not the conventional ladder diagrams since we use two real fields instead of a single complex field. The final result, however, is the same. The correction to the gradient term in Eq. (42) is obtained by summing the same diagrams with insertions of sources $X, Y$, and their derivatives in the leftmost loop in every diagram.

In two dimensions, the summation amounts to replacing the bare interaction $g$ by an effective interaction $-8 \pi\left[\ln \left(\rho a^{2}\right)\right]^{-1}$, which is density dependent $[19,35,36]$. In the dilute-gas limit, this expansion parameter is small, and Eq. (42) is replaced by

$$
\begin{aligned}
0= & {\left[\nabla^{2}+\mu-V\right] \sqrt{\rho}+8 \pi L^{-1}\left\{+(\ln [-L / 8 \pi]-1) L^{-1} \rho \sqrt{\rho}\right.} \\
& +\frac{2}{3} L^{-1} \nabla^{2} \sqrt{\rho},
\end{aligned}
$$


where $L=\left[\ln \left(\rho a^{2}\right)\right]$. This is the main result of the present paper. The second term follows from the ground-state energy of a homogeneous Bose gas. The leading piece of this term, which corresponds to Schick's result, was found in Refs. $[15,16,30]$. The subleading pieces of this term as well as the third term, which is a correction to the gradient term, are our results.

Up to corrections that are suppressed by powers of the small parameter $-8 \pi\left[\ln \left(\rho a^{2}\right)\right]^{-1}$, Eq. (44) can be derived from an energy functional $E[\Phi]$, where $[\Phi]$ is identified with $\sqrt{\rho}$,

$$
\begin{aligned}
E[\Phi]= & \int d^{2} x\left\{\left[1+\frac{2}{3} L^{-1}\right]|\nabla \Phi|^{2}+[V-\mu]|\Phi|^{2}-4 \pi L^{-1}\right. \\
& \left.\times\left\{1+\frac{1}{2}(2 \ln [-L / 8 \pi]-1) L^{-1}\right\}|\Phi|^{4}\right\}
\end{aligned}
$$

The energy functional Eq. (45) generalizes the energy functional in Refs. $[15,30]$ to include a gradient term.

\section{SUMMARY}

In this paper, we have computed the leading quantum corrections to the Gross-Pitaevskii equation for a trapped Bose gas in two spatial dimensions. The method involves the truncation of two systematic expansions. The first is the quantum loop expansion, which is an expansion in powers of the coupling constant $g$, and the second is a gradient expansion around the Thomas-Fermi limit. The result Eq. (42) includes all leading-order quantum corrections to second order in the gradient expansion.

The summation of the ladder diagrams changes the effective expansion parameter from $g$ to $-8 \pi\left[\ln \left(\rho a^{2}\right)\right]^{-1}$. The resulting equation (44) includes a term that can be derived from the ground-state energy density of a homogeneous Bose gas. It also includes a term that is a gradient correction to the mean-field equation.

\section{ACKNOWLEDGMENTS}

The authors would like to thank E. Braaten for useful discussions. This work was supported by the Stichting voor Fundamenteel Onderzoek der Materie (FOM), which is supported by the Nederlandse Organisatie voor Wetenschapplijk Onderzoek (NWO).

\section{APPENDIX A: FORMULAS}

The loop integrals that appear in our calculations involve integrations over the energy $\omega$ and the spatial momentum $\mathbf{k}$. The energy integrals are evaluated using contour integration. The specific integrals needed are

$$
\begin{aligned}
& \int \frac{d \omega}{2 \pi} \frac{1}{\left(\omega^{2}-\epsilon^{2}+i \epsilon\right)^{n}} \\
& \quad=i(-1)^{n+1} \frac{(-1) \times 1 \times 3 \cdots(2 n-3)}{2^{n}(n-1) !} \frac{1}{\epsilon^{2 n-1}},
\end{aligned}
$$

$$
\begin{aligned}
& \int \frac{d \omega}{2 \pi} \frac{\omega^{2}}{\left(\omega^{2}-\epsilon^{2}+i \epsilon\right)^{n+1}} \\
& \quad=i(-1)^{n+1} \frac{(-1) \times 1 \times 3 \cdots(2 n-3)}{2^{n+1} n !} \frac{1}{\epsilon^{2 n-1}} .
\end{aligned}
$$

The momentum integrals are evaluated using dimensional regularization in $d=2-2 \epsilon$ dimensions. Some of the integrals are infrared divergent or ultraviolet divergent or both. They can be written in terms of integral $I_{m, n}$, which is defined by

$$
I_{m, n}=\left(\frac{e^{\gamma} M^{2}}{4 \pi}\right)^{\epsilon} \int \frac{d^{d} k}{(2 \pi)^{d}} \frac{k^{2 m}}{k^{n}\left(k^{2}+\Lambda^{2}\right)^{n / 2}} .
$$

Here, $M$ is a renormalization scale that ensures that $I_{m, n}$ has the canonical dimension also for $d \neq 2 . \quad \gamma \approx 0.5772$ is the Euler-Mascheroni constant. With dimensional regularization, $I_{m, n}$ is given by the formula

$$
\begin{aligned}
I_{m, n}= & \frac{\Omega_{d}}{(2 \pi)^{d}}\left(\frac{e^{\gamma} M^{2}}{4 \pi}\right)^{\epsilon} \Lambda^{d+2 m-2 n} \\
& \times \frac{\Gamma\left(\frac{d-n}{2}+m\right) \Gamma\left(n-m-\frac{d}{2}\right)}{2 \Gamma\left(\frac{n}{2}\right)},
\end{aligned}
$$

where $\Omega_{d}=2 \pi^{d / 2} / \Gamma[d / 2]$ is the area of the $d$-dimensional sphere.

The integrals $I_{m, n}$ satisfy the relations

$$
\begin{gathered}
(d+2 m-n) I_{m, n}=n I_{m+2, n+2}, \\
\Lambda^{2} I_{m, n}=I_{m-1, n-2}-I_{m+1, n} .
\end{gathered}
$$

The first relation follows from integration by parts, while the second is simply an algebraic relation.

\section{APPENDIX B: COEFFICIENTS}

The coefficients needed to calculate $\left\langle\psi_{1}^{2}\right\rangle$ and $\left\langle\psi_{2}^{2}\right\rangle$ in Eqs. (29) and (30) were calculated and listed in Ref. [6]. For completeness, we list them below:

$$
\begin{aligned}
& a_{0}=\frac{1}{2} I_{1,1}, \\
& a_{1}=\frac{1}{4} I_{2,3}, \\
& a_{2}=-\frac{1}{4} I_{0,1}, \\
& a_{3}=\frac{1}{16 d}\left[-10 I_{5,7}+13 I_{3,5}-I_{1,3}\right], \\
& a_{4}=\frac{3}{16} I_{3,5}, \\
& a_{5}=\frac{1}{64 d}\left[35 I_{6,9}-10 I_{4,7}+3 I_{2,5}\right], \\
& b_{0}=\frac{1}{2} I_{-1,-1},
\end{aligned}
$$




$$
\begin{aligned}
& b_{1}=-\frac{1}{4} I_{0,1}, \\
& b_{2}=\frac{1}{4} I_{-2,-1}, \\
& b_{3}=\frac{1}{16 d}\left[2 I_{3,5}-3 I_{1,3}-I_{-1,1}\right], \\
& b_{4}=-\frac{1}{16} I_{1,3}, \\
& b_{5}=-\frac{5}{64 d}\left[I_{4,7}+2 I_{2,5}+I_{0,3}\right] .
\end{aligned}
$$

The factor of $d$ in the denominators in $a_{3}, a_{5}, b_{3}$, and $b_{5}$ arises from averaging over angles in the momentum integrals.
Finally, we outline how to calculate a one-loop diagram explicitly. For simplicity, we compute the leading coefficient $a_{0}$ in the gradient expansion for the expectation value $\left\langle\psi_{1}^{2}\right\rangle$ in Eq. (29). The diagram is shown in Fig. 1, and it reads

$$
a_{0}=\left(\frac{e^{\gamma} M^{2}}{4 \pi}\right)^{\epsilon} \int \frac{d \omega}{2 \pi} \int \frac{d^{d} k}{(2 \pi)^{d}} \frac{i k^{2}}{\omega^{2}-\epsilon^{2}+i \epsilon}
$$

After integrating over the energy $\omega$, using Eq. (A1), we obtain

$$
a_{0}=\frac{1}{2}\left(\frac{e^{\gamma} M^{2}}{4 \pi}\right)^{\epsilon} \int \frac{d^{d} k}{(2 \pi)^{d}} \frac{k^{2}}{\epsilon}=\frac{1}{2} I_{1,1} .
$$

[1] M. H. Anderson, J. R. Ensher, M. R. Matthews, C. E. Wieman, and E. A. Cornell, Science 269, 198 (1995).

[2] K. B. Davis, M. O. Mewes, M. R. Andrews, N. J. van Druten, D. S. Durfee, D. M. Kurn, and W. Ketterle, Phys. Rev. Lett. 75, 3969 (1995).

[3] C. C. Bradley, C. A. Sackett, J. J. Tollett, and R. G. Hulet, Phys. Rev. Lett. 75, 1687 (1995).

[4] F. Dalfovo, S. Giorgini, L. P. Pitaevskii, and S. Stringari, Rev. Mod. Phys. 71, 463 (1999).

[5] S. L. Cornish, N. R. Claussen, J. L. Roberts, E. A. Cornell, and C. E. Wieman, Phys. Rev. Lett. 85, 1795 (2000).

[6] E. Braaten and A. Nieto, Phys. Rev. B 56, 14745 (1998).

[7] J. O. Andersen and E. Braaten, Phys. Rev. A 60, 2330 (1999).

[8] D. Blume and C. H. Greene, Phys. Rev. A 63, 063601 (2001).

[9] M. Schick, Phys. Rev. A 3, 1067 (1971).

[10] V. N. Popov, Theor. Math. Phys. 11, 565 (1972).

[11] V. N. Popov, Functional Integrals in Quantum Field Theory and Statistical Physics (Reidel, Dordrecht, 1983).

[12] D. F. Hines, N. E. Frankel, and D. J. Mitchell, Phys. Lett. 68A, 12 (1978).

[13] D. S. Fisher and P. C. Hohenberg, Phys. Rev. B 37, 4936 (1988).

[14] E. B. Kolomeisky and J. P. Straley, Phys. Rev. B 46, 11749 (1992).

[15] E. H. Lieb and J. Yngvason, e-print math-ph/0002014.

[16] E. H. Lieb, R. Seiringer, and J. Yngvason, e-print cond-mat/0005026.
[17] N. D. Mermin and H. Wagner, Phys. Rev. Lett. 22, 1133 (1966).

[18] P. C. Hohenberg, Phys. Rev. 158, 383 (1967).

[19] J. M. Kosterlitz and D. J. Thouless, J. Phys. C 6, 1181 (1973).

[20] A. I. Safonov, S. A. Vasilyev, I. S. Yasnikov, I. I. Lukashevich, and S. Jaakkola, Phys. Rev. Lett. 81, 4545 (1998).

[21] V. Bagnato and D. Kleppner, Phys. Rev. A 44, 7439 (1991).

[22] T-L. Ho and M. Ma, J. Low Temp. Phys. 115, 61 (1999).

[23] D. S. Petrov, M. Holzmann, and G. V. Shlyapnikov, Phys. Rev. Lett. 84, 2551 (2000).

[24] W. J. Mullin, J. Low Temp. Phys. 106, 615 (1997).

[25] A. Görlitz, J. M. Vogels, A. E. Leanhardt, C. Raman, T. L. Gustavson, J. R. Abo-Shaeer, A. P. Chikkatur, S. Gupta, S. Inouye, T. P. Rosenband, D. E. Pritchard, and W. Ketterle, e-print cond-mat/0104549.

[26] E. A. Hinds et al., Phys. Rev. Lett. 80, 645 (1998).

[27] T. Haugset and H. Haugerud, Phys. Rev. A 57, 3809 (1998).

[28] S. K. Adhikari, Phys. Rev. Lett. 265, 91 (2000).

[29] S. K. Adhikari, Phys. Rev. E 62, 2937 (2000).

[30] E. B. Kolomeisky, T. J. Newman, J. P. Straley, and X. Qi, Phys. Rev. Lett. 85, 1146 (2000).

[31] E. Braaten, H.-W. Hammer, and S. Hermans, Phys. Rev. A 63, 063609 (2001).

[32] O. Bergman, Phys. Rev. D 46, 5474 (1992).

[33] G. Lozano, Phys. Lett. B 283, 70 (1992).

[34] T. Haugset and F. Ravndal, Phys. Rev. D 49, 4299 (1994).

[35] V. N. Popov, Functional Integrals and Collective Excitations (Cambridge University Press, Cambridge, England, 1987).

[36] H. T. C. Stoof and M. Bijlsma, Phys. Rev. E 47, 939 (1993). 